\title{
man \\ Blending of Hydrothermal Liquefaction Biocrude with Residual Marine Fuel: An Experimental Assessment
}

\author{
Andrea Maria Rizzo ${ }^{1,2}$ (D) and David Chiaramonti $1,3, *$ (D) \\ 1 Renewable Energy Consortium for Research and Demonstration (RE-CORD), Scarperia e San Piero, \\ 50038 Florence, Italy; andreamaria.rizzo@re-cord.org \\ 2 Department of Industrial Engineering, University of Florence, 50134 Florence, Italy \\ 3 Department of Energy “Galileo Ferraris", Polytechnic of Turin, 10129 Turin, Italy \\ * Correspondence: david.chiaramonti@polito.it
}

check for updates

Citation: Rizzo, A.M.; Chiaramonti, D. Blending of Hydrothermal Liquefaction Biocrude with Residual Marine Fuel: An Experimental Assessment. Energies 2022, 15, 450. https://doi.org/10.3390/ en15020450

Academic Editor: Mariusz J. Stolarski

Received: 1 November 2021

Accepted: 1 January 2022

Published: 10 January 2022

Publisher's Note: MDPI stays neutral with regard to jurisdictional claims in published maps and institutional affiliations.

Copyright: (C) 2022 by the authors. Licensee MDPI, Basel, Switzerland. This article is an open access article distributed under the terms and conditions of the Creative Commons Attribution (CC BY) license (https:// creativecommons.org/licenses/by/ $4.0 /)$.
Abstract: As with all transport modes, the maritime sector is undergoing a drastic transition towards net zero, similar to the path in which Aviation is already engaged through global decarbonization programs such as CORSIA for the International Civil Aviation Organization, or the Emission trading Scheme of the European Union). Maritime indeed shares with Aviation a common element: the difficulty of shifting to electric in the short to medium term. Therefore, the use of sustainable fuels represents the main and only relevant option in this timeframe. As sustainable biofuels will be used as blend components in the case of large-scale deployment, it is necessary to investigate the behavior of bio- and fossil-based fuels when mixed in various percentages, in particular for low quality products such as HTL (HydroThermal Liquefaction) and fast pyrolysis oils from lignocellulosic biomass and waste. Biocrude from subcritical hydrothermal liquefaction of undigested sewage sludge, produced at reaction conditions of $350{ }^{\circ} \mathrm{C}$ and 200 bar in a continuous HTL pilot scale unit, was manually mixed at $70^{\circ} \mathrm{C}$ with residual marine fuel (low-sulphur type F-RMG-380 per ISO 8217) at two different nominal biocrude shares, respectively $10 \mathrm{wt} . \%$ and $20 \mathrm{wt} . \%$ in the mixture. While the former blend resulted in the technically complete dissolution of biocrude in the fossil component, the latter sample formed biocrude agglomerates and only partial dissolution of the biocrude aliquot in marine fuel could be achieved (calculated between 14-16 wt.\%). The blend with $10 \mathrm{wt} . \%$ of SS biocrude in the mixture resulted in compliance with limits of total acid number (TAN), inorganics (in particular vanadium, sodium, silicon and aluminum) and sulphur content, while only the ash content was slightly above the limit.

Keywords: biocrude; marine fuel; blend

\section{Introduction}

Maritime transport is an essential component of the European Union's (EU) transport system both for goods and for the mobility of EU citizens. It contributed to, respectively, around $75 \%$ and $31 \%$ of EU external and internal trade volume, and around 400 million passengers embarked or disembarked in EU ports in 2019 [1,2]. The European Commission set the ambitious goals of reducing greenhouse gas (GHG) emission by $55 \%$ by 2030 , and to become carbon neutral by 2050 [3]. To achieve these results, a reduction of $90 \%$ in transport emission is required by 2050 and, based on the policy scenarios reported in the Climate Target Plan (CTP), renewable and low carbon fuels should represent between 6\% to $9 \%$ of the international maritime transport fuel mix by 2030 and up to $88 \%$ by 2050 , so as to achieve the expected GHG emission reduction target [1].

Hydrothermal liquefaction is one of the most promising technologies under development for the production of sustainable fuels. The process takes place in an aqueous environment at $250-450{ }^{\circ} \mathrm{C}$ and pressure in the range of $10-35 \mathrm{MPa}$. The peculiar properties of water in these conditions promotes the depolymerization of the organic material that 
undergoes several reactions, which include decarboxylation, dehydration and hydrolysis. As a consequence of the process, organic matter is converted in four phases: the biocrude, which is accounted as the main product and is a generally a heavy oil; an aqueous phase, produced in large volumes with dissolved organics in low concentration; a solid residue; and a gas phase mainly composed of $\mathrm{CO}_{2}[4,5]$. This crude product could, in principle, well match the heavy nature of most used fossil-based marine fuels, thus being the ideal candidate for adoption in the sector. On the other hand, marine fuels are the cheapest fuels on the market, and shifting to a renewable fuel is particularly challenging from an economic point of view.

Compared to other thermochemical processes such as pyrolysis or gasification, since HTL occurs in a water-rich medium, does not require a drying step for the feedstock, and for this reason has gained traction for the valorization of wet biomasses [6,7]. Among these, sewage sludge (SS) can be an interesting low cost, renewable and abundant feedstock, not requiring land for its production. Recently, the UN confirmed that the world's population will continue to grow, with a projection of 8.5 billion in 2030 and 9.7 billion in 2050 [8].

The introduction of Directive 91/271/EEC constituted a turning point for the wastewater treatment sector, as pursuing its compliance led to the construction of new wastewater treatment plant across EU, which ultimately translated in significant increase of sludge production during the nineties $(+43 \%)$. Nowadays, however, the trend of sludge generation tended to plateau in almost all the European countries [9]. For the EU-28, Campo et al. [9] estimated that the annual SS production in 2017 was in the order of 9.0-9.5 million tons of dry substance, though the authors reported that the data were "incomplete and quite fragmented". For Italy alone, the municipal raw SS production in 2019 was 3.4 million tons, only $41 \%$ of which were recycled (agriculture uses and composting) and just $0.9 \%$ used for energy generation [10]. As a consequence of the abundance of this site-concentrated, readily available, wet organic feedstock, a significant amount of research works focused on the conversion of SS through the HTL process for biofuels production, and the most recent are reported here.

Shah et al. investigated the effects of $\mathrm{K}_{2} \mathrm{CO}_{3}$ addition in sub- and supercritical HTL of SS. Their work showed that the addition of $\mathrm{K}_{2} \mathrm{CO}_{3}$ reduced the nitrogen content in the biocrude, in particular under subcritical conditions, while temperature increases from $350{ }^{\circ} \mathrm{C}$ to $400{ }^{\circ} \mathrm{C}$ had no influence on biocrude yield; the maximum biocrude (BC) yields were obtained at $350{ }^{\circ} \mathrm{C}(45.22 \mathrm{wt} . \%)$ [11]. The same reaction temperature was suggested by $\mathrm{L}$. Qian et al. as the optimal condition to maximize the BC yield, even if the attained yield was considerably lower, being $30.9 \mathrm{wt} . \%$ [12].

Biocrude from HTL of SS is a heterogeneous oxygenated mixture that contains a high concentration of nitrogen and oxygen-based heterocyclic compounds $[11,13,14]$ and a further upgrade step is necessary to upgrade it into a transportation fuel. Hydrotreatment is a possible pathway for its valorization and consists in the reaction of biocrude with $\mathrm{H}_{2}$ at high pressure in the presence of a catalyst [15], which, however, adds significant costs and complexity.

Castello et al. and Jarvis et al. investigated the potential of SS conversion in HTL and subsequent upgrading to drop-in biofuel. Castello et al. reported a remarkable production of straight chain paraffinic hydrocarbons in the light diesel range through the upgrade of SS biocrude using commercial $\mathrm{NiMo} / \mathrm{Al}_{2} \mathrm{O}_{3}$ catalyst [16]. Jarvis et al. analyzed in details the composition of raw and hydrotreated SS biocrude, and reported that hydrotreated sample was mainly composed of hydrocarbon species (almost 40\%), suggesting that raw biocrude undergoes deoxygenation and denitrogenation reactions [17].

Compared to full hydrotreating, a lower cost approach to the introduction of biogenic carbon from SS BC in transportation fuel is co-processing along with fossil crude oil in co-distillation; Chiaberge et al. subjected an SS BC sample to partial hydrotreating to reduce acidity (TAN [total acid number] below $2 \mathrm{mg} \mathrm{KOH} / \mathrm{g}$ from $>100 \mathrm{mg} \mathrm{KOH} / \mathrm{g}$ ), inorganics and $\mathrm{O}_{2}$, and then co-distilled the partially upgraded biocrude at a blending ratio of $20 \%$ with a low acidity fossil crude oil, obtaining four distilled fractions [18]. 
All the previously reported experiences aiming either at the production of a biofuel from the direct upgrading of SS BC or at the introduction of biogenic components from SS BC into refinery streams relied on mild or severe upgrading of SS BC with the use of hydrogen; to the best of our knowledge, the direct introduction of BC SS components into a fossil fuel for marine application without prior upgrading has not been reported yet.

The annual global marine fuel consumption accounts for more than 400 million tons, and it is dominated by heavy fuel oil, also called residual marine fuel (MF), and to a lower extent, by distillate marine fuel, also called marine gas oil (MGO) $[19,20]$. The former is the residue of the distillation process of crude oil and it is the most-used fuel in shipping due to its low cost, while the latter is a lighter fraction of crude oil that evaporates under fractional distillation [20]. MF are mainly used in medium and low-speed marine diesel engines, while MGO is used in smaller medium- to high-speed units [21-24].

The specification framework for marine fuels is laid down within ISO 8217:2017, where ten grades of residual marine fuel (RMA, RMB, RMD, RME, RMG, RMK) and seven grades of distillate marine fuel (DMX, DMA, DFA, DMZ, DFZ, DMB, DFB) [19,25] are identified and regulated. MF are also classified in high-, medium- or low-sulphur (S) fuel and according to ISO 8217, and the quantity of S should not exceed $3.5 \mathrm{wt} . \%$ [22].

The maritime sector significantly contributes to air pollution in coastal areas, and the particulate matter $(\mathrm{PM})$ and sulphur oxides emissions are particularly relevant in this context. The ocean-going ships emit 1.2-1.6 million metric tons of PM and 4.7-6.5 million metric tons of $\mathrm{SO}_{x}$ per year, causing serious problems for public health in particular in highly populated coastal areas [26]. For these reasons, the Council Directive EU 2016/802 laid down the maximum permitted sulphur content in heavy fuel oil, gas oil, marine gas oil and marine diesel oil used in the EU. It imposed that the Members States should ensure a maximum sulphur content in marine fuel used in their territory of $3.5 \mathrm{wt} . \%$. The only exception is for ships with emission abatement methods that allow them to reduce the sulphur dioxide emission to the same level achieved by using marine fuels with low sulphur content [27]. The International Maritime Organization made the decision to further reduce the limit of the sulphur content in marine fuel to $0.5 \mathrm{wt} . \%$ as of 1 January 2020. As a consequence, the demand for low sulphur fuel will globally increase, and the expected drop in overall $\mathrm{SO}_{\mathrm{x}}$ emission from ships will be $77 \%$ [28]. The Marine Environment Protection Committee has estimated that a delay in implementing this legislation on low sulphur marine fuel from 2020 to 2025 would contribute to more than 570,000 additional premature deaths [29].

The aim of the present work is to investigate the possibility of blending a biocrude obtained from the HTL of SS with a sample of residual marine fuel (MF, grade ISO-FRMG 380). The experimental study was mainly focused to verify the actual mixing of the two components, fossil and bio-based, at different biocrude nominal concentrations (10 wt.\% and $20 \mathrm{wt} . \%$ ) using analytical methods (elemental analysis, FT-IR). The influence of biocrude concentration in blend was also assessed against the relevant MF standard.

To the best of our knowledge, this is the first reported attempt at producing a low-cost direct blend between HTL biocrude from sewage sludge and ISO-F-RMG 380 residual marine fuel, without applying any upgrading step, that yields a fuel blend compliant with a selection of basic quality parameters set forth by the applicable marine fuel standard (ISO 8217).

\section{Materials and Methods}

\subsection{Raw Materials}

Sewage sludge was obtained from an Italian municipal waste-water treatment plant (WWTP). The sample was collected before anaerobic digestion (it was only subject to aerobic stabilization) and provided without drying.

Residual marine fuel (MF) compliant with ISO 8217:2017 for the category ISO-F-RMG 380 was obtained from Eni S.p.a. MF is produced from various refinery streams, usually residual ones; the composition is complex, varying with the sources of crude oil $[30,31]$. 


\subsection{Hydrothermal Liquefaction Experiment}

The continuous HTL plant of RE-CORD is able to process $1-21 \mathrm{~h}^{-1}$ of inlet slurry and a detailed description of the plant (Figure 1) has already been published [32]. Municipal SS was dried in an oven at $105^{\circ} \mathrm{C}$ and then milled with deionized water (DI) water to obtain a biomass-to-water (B/W) ratio of $10 \mathrm{wt} . \%$. The HTL experiment was performed at nominal reaction conditions set at $350{ }^{\circ} \mathrm{C}$ and 200 bar. Before the experiment, water was pumped into the system at the design pressure to heat-up the plant. Once steady temperatures were reached, the slurry was fed. Products were filtered in a $100 \mu \mathrm{m}$ wire-mesh and then collected in a tank.

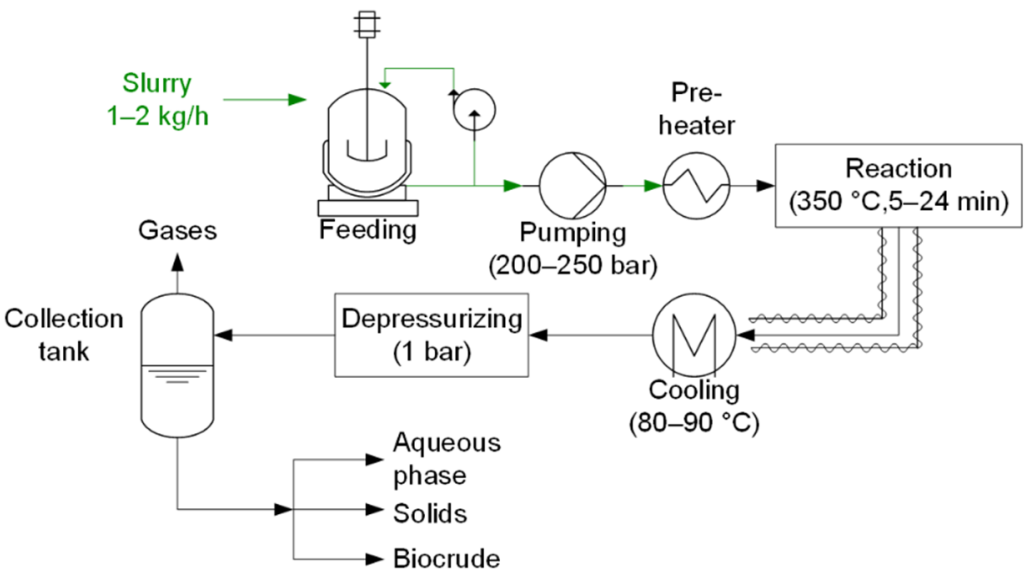

Figure 1. Simplified flow chart of RE-CORD HTL plant.

At the end of the test, water was pumped to cool down the system and allow depressurization, then acetone (dimethyl ketone-DMK) was pumped into the plant to recover the residual biocrude deposited in the pipes. Moreover, a thorough cleaning with alkaline solution was performed to clean the internal surface of the plant. A small amount of biocrude and solids remained deposited in internal surface of the depressurization system, and they were recovered before DMK washing.

Biocrude and solids were gravimetrically separated from the liquid phase and the former were washed with DMK and vacuum-filtered at $1 \mu \mathrm{m}$ over a Whatman glass microfiber filter. The DMK-solubles were then rotary evaporated at $60^{\circ} \mathrm{C}, 50 \mathrm{kPa}, 60 \mathrm{rpm}$ to recover the biocrude. The filter with the solid residue (SR) was oven-dried at $105^{\circ} \mathrm{C}$ overnight and weighed. A small quantity of solids and biocrude remained dispersed in the liquid phase after gravimetric separation and, consequently, the latter was subjected to the same aforementioned procedure for biocrude and solids collection. The DMK collected during plant washing was vacuum filtered and subjected to rotary evaporation. The products collected from the pistons of the depressurization system were recovered using the same procedure described for the liquid phase. Figure 2 reports a scheme of the extraction procedure for the separation of HTL products. The biocrude used in this work was recovered through DMK washing after gravimetrically separation of the liquid phase. 


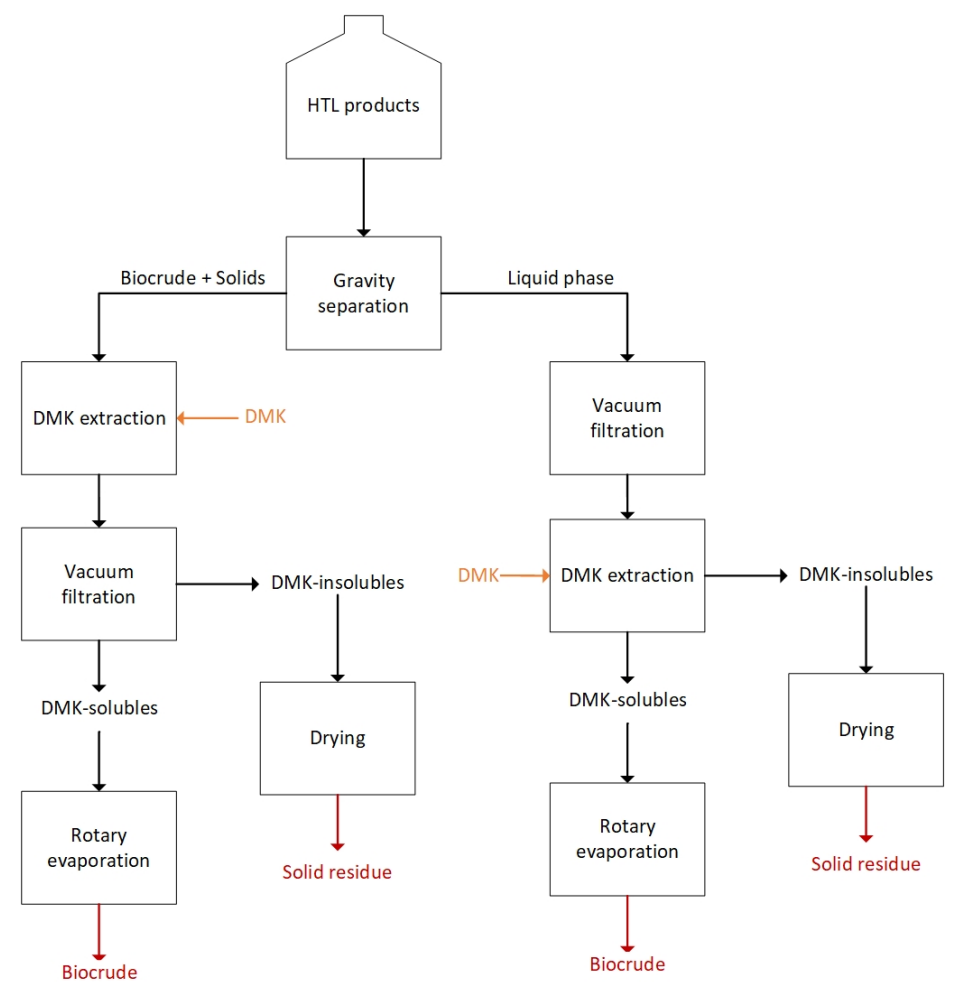

Figure 2. Extraction procedure for HTL products recovery.

\subsection{Analytical Methods and Chemicals}

ACS reagent-grade solvents and reagents were obtained from Carlo Erba and SigmaAldrich and used as received.

Proximate analyses were carried out in a Leco TGA 701 according to EN ISO 18134. The content of hydrogen, nitrogen, sulphur and carbon in biocrudes, marine fuel and blends were measured in a Leco TruSpec CHN-S analyser according to UNI EN 16948 and ASTM D4239, while the oxygen content was measured according DIN 51732:2014.

FT-IR spectra were acquired from 4000 to $600 \mathrm{~cm}^{-1}$, with a $4 \mathrm{~cm}^{-1}$ resolution, using a Shimadzu IRTracer-100 equipped with QATR ${ }^{\mathrm{TM}} 10$ Single-Reflection ATR and a diamond crystal.

The content of inorganics was performed by an Agilent ICP-OES A200 MP-AES after sample digestion in a microwave oven (Milestone Start D) according to UNI EN ISO 16967-16968.

The total acid number of the blend samples was measured according to DIN EN 12634: 1999 using a non-aqueous potentiometric titration method developed for petroleum products and lubricants.

The two blends (10 wt.\% and 20 wt.\% concentration of BC) and the MF were analysed with an optical microscope at $10 \times$ magnification.

Biocrude's apparent molar mass was determined in triplicate as polystyrene-equivalent by means of gel permeation chromatography (GPC). Analyte was dissolved in tetrahydrofuran (THF), left overnight, and then filtered at $0.45 \mu \mathrm{m}$. Then, $100 \mu \mathrm{L}$ of the solution was injected in HPLC (Shimadzu LC 20 AT Prominence) connected to a refractive index detector (RID) fitted with two columns (Agilent, PL gel $5 \mu \mathrm{m} 100 \AA 300 \times 7.5 \mathrm{~mm}$ ) plus a guard (Agilent, PL gel $5 \mu \mathrm{m} 50 \times 7.5 \mathrm{~mm}$ ) at $40{ }^{\circ} \mathrm{C}$ under $1 \mathrm{~mL} / \mathrm{min}$ of THF as eluent. The system's calibration used polystyrene standards from Agilent with molecular weights in the range 370-9960 $\mathrm{g} / \mathrm{mol}$.

Data of carbon, hydrogen and nitrogen content of MF, Blend A, Blend B, Blend B residue and SS biocrude were processed with a one-way analysis of variance (ANOVA) followed by Tukey test at 99\% confidence level (Minitab ${ }^{\circledR}$ 18.1, Minitab Inc., State College, PA, USA). The normality and homogeneity of the parameters were tested prior to ANOVA. 
Whenever possible, analytical determinations have been carried out at least in duplicate, and the associated standard deviations have been reported between brackets, next to the measured value.

\subsection{Blending Tests}

Two blending experiments were performed by manually mixing sewage sludge (SS) biocrude and residual marine fuel (MF) oil in a $50 \mathrm{~mL}$ beaker under continuous stirring in a $70{ }^{\circ} \mathrm{C}$ thermostatic bath.

In the first experiment, $2 \mathrm{~g}$ of SS biocrude was mixed with $18 \mathrm{~g}$ of MF to give a nominal $10 \mathrm{wt} . \%$ biocrude concentration in blend (Blend A), while a second sample with a nominal biocrude content of $20 \mathrm{wt}$.\% (Blend B) was prepared by mixing $3 \mathrm{~g}$ of SS BC with $12 \mathrm{~g}$ of MF.

\subsection{Calculation of Blending Level}

The appraisal of the actual amount of biocrude in the blend in case of uncomplete mixing was calculated separately from the measured carbon and nitrogen contents of the blend, marine fuel and biocrude, as per Equation (1):

$$
\zeta_{i, \text { blend }}=\chi_{\mathrm{BC}} \cdot \zeta_{i, \mathrm{BC}}+\left(1-\chi_{\mathrm{BC}}\right) \cdot \zeta_{i, \mathrm{MF}}
$$

where $i$ is the $i$-th element $(\mathrm{C}, \mathrm{N}), \zeta_{i, \ldots}$ is the concentration of the $i$-th element, respectively, in the blend, biocrude and marine fuel, and $\chi_{\mathrm{BC}}$ is the real amount of biocrude in blend.

\section{Results and Discussion}

\subsection{Biocrude and Marine Fuel Analyses}

The choice of the analyses to be performed was mandated primarily by the limited amount of biocrude available, which prevented the possibility of making extensive physical measurements to characterize its properties, and secondarily by the relevance to the compliance of the blend to the fuel specification. The selected analyses were the elemental composition, acidity (as TAN), vanadium, sodium and ashes, and the rationale underpinning their choice is more extensively detailed in the following paragraphs.

The elemental composition was measured because the relative abundances of $\mathrm{C}, \mathrm{H}, \mathrm{O}$, $\mathrm{S}$, and $\mathrm{N}$ for the biocrude and the marine fuel are peculiar and distinct. The biocrude is oxygenated and is high in nitrogen and therefore lower in carbon and hydrogen compared to the marine fuel, which, on the contrary, has practically no oxygen and a limited amount of nitrogen and sulphur. Assuming that the elemental composition of a blend between the biocrude and the marine fuel is the weight-average of their respective components, from the determination of the elemental composition of the mixture one can calculate the actual amount of biocrude in blend, even in the case of incomplete dissolution of the biocrude, under the hypothesis that all the biocrude's constituents contribute equally to the formation of the blend.

The content of ashes was measured as they are critical elements in fuel applications and can lead to the formation of deposits in combustion. Besides, SS being usually very rich in ashes (>>10 wt.\% dry base), it is common also for the BC obtained from SS liquefaction to present an ash content far higher than the current limit for F-RMG-380, which is $0.1 \mathrm{wt} . \%$.

The acidity was measured because it is known from literature that SS BC tends to have a high acid value, with reported figures comprised between $6.29 \mathrm{mg} \mathrm{KOH} / \mathrm{g}$ [33] as well as above $100 \mathrm{mg} \mathrm{KOH} / \mathrm{g}$ [18], but that in every case exceeds the limit for residual marine fuels $(2.5 \mathrm{mg} \mathrm{KOH} / \mathrm{g})$.

Vanadium, sodium, silicon and aluminum have been analyzed as the amount of these elements are specifically regulated by ISO 8217 for their relevance in combustion applications.

Viscosity, flash point and pour point, among others, also have important and practical implications on the suitability of a fuel for a certain application. Although viscosity could not be directly measured, the biocrude used in the present study was free-flowing at room temperature, and therefore within the reported range for expected dynamic viscosity 
at $40{ }^{\circ} \mathrm{C}$ for a biocrude obtained from sewage sludge under comparable process conditions are comprised $3.39 \mathrm{cP}$ (Rahman et al. [33], for a density of $1.13 \mathrm{~g} / \mathrm{cm}^{3}$ ) and $571 \mathrm{cP}$ (Jarvis et al. [17]). Flash point and pour point could not be analyzed for the biocrude under consideration, and to the best of our knowledge, no results for these two analyses on raw (not upgraded) SS BC have been previously published.

In terms of proximate analysis, the raw sewage sludge had a moisture content of 71.2 wt. $\%$ w.b. and ash content of 19.8 wt. $\%$. Its elemental composition was 57.2 wt. $\%$ carbon, $8.1 \mathrm{wt} . \%$ hydrogen, $5.4 \mathrm{wt} . \%$ nitrogen, $0.7 \mathrm{wt} . \%$ sulphur and $9 \mathrm{wt} . \%$ oxygen on a dry ash free (d.a.f.) basis. The main inorganic elements were (in order of decreasing abundance) Ca (11066 ppm), Al (7794 ppm), Fe (5583 ppm) and P (5398 ppm).

The elemental composition of SS is highly dependent on the collection site. Shah et al. reported, for secondary sewage sludge collected from WWTP (Aalborg Forsyning), 50.95 wt.\% of $\mathrm{C}, 7.36 \mathrm{wt} . \% \mathrm{H}, 6.91 \mathrm{wt} . \% \mathrm{~N}$ on a d.a.f. basis, while $\mathrm{Xu}$ et al. indicated 52.5 wt. \% C, 8.0 wt.\% H, $7.8 \mathrm{wt} . \% \mathrm{~N}$ for SS collected in WWTP in Xi'an city (China) [11,14]. Moreover, the ash composition can be very diversified but some of the main inorganic elements in SS were Ca, P, Al, Fe [11,13,34], as in our sample.

The biocrude was also subjected to gel permeation chromatography for the determination of the apparent molar mass and its distribution. The SS biocrude presents a weight-average molecular weight $(\mathrm{Mw})$ of $601 \mathrm{~g} \mathrm{~mol}^{-1}$ with a polydispersity index (PDI) of 1.5 , indicating a medium breadth in the molecular weight distribution with a compresence of light monomers, unreacted material, and products from condensation/polymerization of intermediate reactions compounds [35-37].

The elemental composition of the marine fuel was determined along with the content in ash and inorganics ( $\mathrm{Va}, \mathrm{Na})$, acidity; results are all reported in Table 1 along with those of biocrude.

Table 1. Chemical characteristics of MF and SS biocrude. Standard deviations, where available, are reported in brackets.

\begin{tabular}{ccccc}
\hline & MF & BC & U.M. & Norm \\
\hline $\mathrm{C}$ & $87.1(0.36)$ & $75.7(0.38)$ & wt. $\%$ & UNI EN ISO 16948 \\
$\mathrm{H}$ & $12.1(0.16)$ & $9.1(0.04)$ & wt. $\%$ & UNI EN ISO 16948 \\
$\mathrm{N}$ & $0.2(0.02)$ & $4.6(0.03)$ & $\mathrm{wt.} \%$ & UNI EN ISO 16948 \\
$\mathrm{O}$ & $<0.5$ & $10.1(0.56)$ & $\mathrm{wt.} \%$ & DIN 51732:2014 \\
$\mathrm{S}$ & $0.38^{\mathrm{a}}$ & $0.98(0.01)$ & $\mathrm{wt} \%$ & UNI EN ISO 14596:2008 \\
Ash & $0.005^{\mathrm{a}}$ & n.a. & wt. $\%$ & UNI EN ISO 6245:2005 \\
TAN & $0.32^{\mathrm{a}}$ & n.a. & $\mathrm{mg} \mathrm{KOH} / \mathrm{g}$ & ASTM D664-18e2 \\
Vanadium & $6^{\mathrm{a}}$ & $1(0.05)$ & $\mathrm{mg} / \mathrm{kg}$ & $\mathrm{IP} \mathrm{501/05}$ \\
Sodium & $1^{\mathrm{a}}$ & $36(0.25)$ & $\mathrm{mg} / \mathrm{kg}$ & $\mathrm{IP} \mathrm{501/05}$ \\
\hline
\end{tabular}

a: Analysis provided by the supplier along with the sample.

\subsection{Blending Tests}

Blend A resulted in a homogeneous, mobile black liquid; the presence of suspended particles or agglomerates could not be detected, suggesting that most of the biocrude was dissolved in the MF. To gather confirmation of the apparent homogeneity, samples of the blend were taken at multiple heights of the beaker and separately analyzed (see Section 3.3).

For Blend B, notwithstanding the thorough mixing of biocrude and MF, the formation of agglomerates could instead be clearly identified; to obtain a homogeneous sample and separate the agglomerates, the blend was filtered using a $1 \mathrm{~mm}$ sieve that retained the larger solid particles, yielding almost $1 \mathrm{~g}$ of material. Both phases were analyzed to obtain their elemental composition and inorganics content.

Homogeneous samples of Blend A and B were placed under optical microscope and observed at 10x magnifications; no clear differences could be visually established between Blend A and B, as shown in Figure 3, where an optical image of pristine MF (a) is reported along with those of Blend A (b) and Blend B (c). While MF is a completely homogeneous phase, the addition of the SS biocrude resulted in the formation of what 
seem to be agglomerates dispersed in the continuous oily phase (the MF) for both Blend A and B cases.

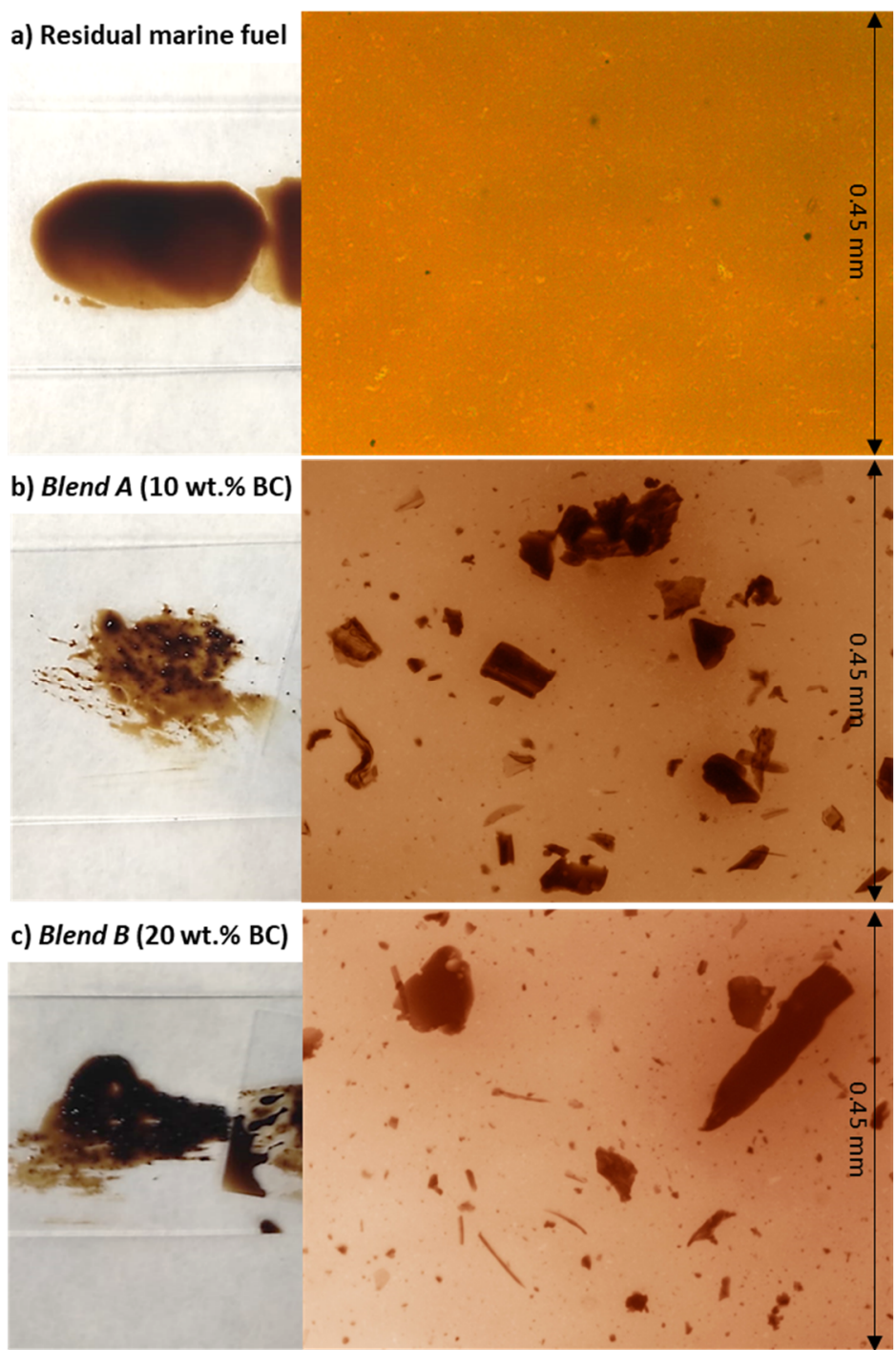

Figure 3. Images obtained with optical microscope at 10x magnification of: (a) MF, (b) Blend A, (c) Blend B (BC = biocrude from HTL of SS).

Particles size for the agglomerates found in both mixtures were measured using the optical microscope software and a graduated slide. For both blends, the particle dimension resulted to be well below 50-100 $\mu \mathrm{m}$, suggesting similar dissolution of SS biocrude in MF irrespective of its concentration.

Image microscopy is a qualitative technique aimed at giving a glimpse on the effectiveness of the dispersion of biocrude in marine fuel for both samples at nominal concentration of 10 and $20 \%$, which resulted to be visually comparable in terms of presence of micron-size agglomerates distributed in the continuous phase.

Based on the optical microscopy image reported earlier, and without relying on more sophisticated techniques (e.g., laser scattering) to quantitatively assess the quality of the mixing between, for example in terms of size distribution of the droplets of biocrude in the continuous phase (the marine fuel), nor in more energy-intensive mixing techniques, the authors stipulate that manual mixing of a viscous biofuels, itself having a highly complex and articulated composition with compounds of different polarities, can lead to the formation of droplets of irregular size when mixed with marine fuel. The use of 
high-shear techniques is therefore suggested to overcome this limitation when forming the mixture.

\subsection{Analytical Determinations on Blends}

The results of CHNO analysis (Table 2) corroborate the actual presence of biocrude in both blends. Indeed, concurrent decrease in the carbon content and increase of the nitrogen and oxygen contents could be measured for both blends, a result that is consistent with the weight-averaged contribution of biocrude presence into the blend. Moreover, the one-way analysis of variance followed by Tukey test confirmed that there was a statistically significant difference $(p$-value $\leq 0.01)$ between the values of carbon and nitrogen content of Blend $\mathrm{A}$ and MF, while hydrogen values showed no significant differences.

Table 2. Carbon, hydrogen, nitrogen and oxygen content of SS biocrude, MF and blend samples (Blend A, B, and B residue). Values are the means $(n=3)$ with corresponding standard deviation between brakets. Statistically significant differences $(p<0.01)$ between mean values are shown with distinct letters. Capital letters $(\mathrm{A}, \mathrm{B})$ indicate statistically significant differences between $\mathrm{SS}$ biocrude and Blend $B$ residue, lower-case letters $\left({ }^{\mathrm{a}-\mathrm{c}}\right)$ indicate statistically-significant differences between $\mathrm{MF}$, Blend $\mathrm{A}$ and Blend B.

\begin{tabular}{cccccc}
\hline Sample & C wt.\% & H wt.\% & N wt.\% & O wt.\% & S wt.\% \\
\hline SS biocrude & $75.7(0.38)^{\mathrm{A}}$ & $9.1(0.04)^{\mathrm{B}}$ & $4.6(0.03)^{\mathrm{A}}$ & $10.1(0.56)$ & $0.98(0.01)$ \\
Blend B residue & $77.3(1.12)^{\mathrm{A}}$ & $9.6(0.22)^{\mathrm{A}}$ & $3.6(0.10)^{\mathrm{B}}$ & n.d. & n.d. \\
MF & $87.1(0.36)^{\mathrm{a}}$ & $12.1(0.16)^{\mathrm{a}}$ & $0.2(0.02)^{\mathrm{c}}$ & $<0.5$ & 0.38 \\
Blend A & $86.0(0.10)^{\mathrm{b}}$ & $12.0(0.13)^{\mathrm{a}}$ & $0.5(0.08)^{\mathrm{b}}$ & $<0.5$ & $0.47(0.005)$ \\
Blend B & $85.2(0.26)^{\mathrm{b}}$ & $12.0(0.10)^{\mathrm{a}}$ & $0.79(0.04)^{\mathrm{a}}$ & $0.9(0.09)$ & n.d. \\
\hline
\end{tabular}

n.d.: not determined.

The solid residue of Blend B was instead comparable, in terms of elemental analysis, to the SS biocrude, although exhibiting a lower nitrogen content $(3.6 \mathrm{wt} . \%$ compared to $4.6 \mathrm{wt} . \%$ ); in addition, ANOVA results confirmed that there was not a statistically significant difference between the carbon contents of the residue separated from Blend B and SS biocrude (Table 2).

Given the fact that for Blend B not all the SS biocrude was effectively dissolved in the $\mathrm{MF}$, and the elemental compositions of biocrude and MF are significantly distinct, with biocrude exhibiting higher $\mathrm{N}$ and $\mathrm{O}$ contents and lower $\mathrm{C}$ content, the actual biocrude content in Blend B was calculated from its elemental composition, as weight average of the $\mathrm{BC}$ and MF compositions for their respective abundance in blend.

Analyzing the elemental composition of Blend B after filtration, and with reference to Equation (1), the measured values confirmed that only part of the SS biocrude was dissolved in the MF. In particular, the reduction of carbon content compared to the MF sample suggested that only $16 \mathrm{wt} . \%$ of the biocrude was effectively dissolved in the MF, while the increase in nitrogen content indicated the dissolution of $14 \mathrm{wt} . \%$ of biocrude. From a statistical point of view, the ANOVA analysis showed no significant differences between the carbon content of Blend A and Blend B, supporting the conclusion that only part of the biocrude was dissolved in MF in the case of Blend B (Table 2).

These mixing experiments therefore suggest that the blending limit of SS biocrude in MF is comprised between 14-16 wt.\%. A further evidence of the presence of biocrude in the blend is supported by the increased oxygen content compared to the MF sample (Table 2); however, it is difficult to evaluate the biocrude dissolution through this result because the oxygen content in MF was below the calibration limit of the measuring instrument. Finally, the hydrogen content of both blends remained almost unchanged from that of MF, therefore, due to the limited variations in hydrogen content from the baseline value, the application of Equation (1) from this specific element was not meaningful.

Nitrogen is not absent in petroleum products, and its presence in crude oil is generally below $1 \mathrm{wt} . \%$ as heterocyclic compounds such as pyridines (basic nitrogen), pyrroles (non- 
basic nitrogen) [38] as well as primary amines $\left(\mathrm{R}^{-} \mathrm{NH}_{2}\right)$ [39], which have been reported also in qualitative and quantitative compositional analyses on SS biocrude.

Zhai et al. [40] reported that nitrogen-containing species in SS BC were mainly pyridines, amines, indoles, amides, imidazoles and nitrile. Similar nitrogen-containing species were detected by Shah et al. [11] in SS biocrude; there, the main components were amides and nitrogen-heterocyclic compounds, including Pyrimidine-2-methyl, 2(1H)Pyridinone-1-propyl, Pyrazine-2-ethyl-3-methyl, Indole, and its derivatives.

Unlike other biocrudes from lignocellulosic biomasses, the nitrogen-containing compounds found in biocrude samples from the hydrothermal processing of sewage sludge share closer similarities with those identified in fossil crudes, thus are likely miscible in the residual marine fuel used for the present tests as they are already present in fossil crude oils. The affinity of biocrude $\mathrm{N}$-containing compounds with MF was confirmed by the statistically significant increase of nitrogen content between Blend A and Blend B (Table 2) and by the FT-IR analysis performed on both SS biocrude and the two blends described in Section 3.6.

The main inorganic elements measured in the SS biocrude were Fe (716 ppm), Ca (202 ppm), P (196 ppm), K (106 ppm) and Si (90 ppm). The observed concentrations of inorganics in the agglomerates separated from Blend $B$ in the $1 \mathrm{~mm}$ sieve (Table 3 ) were higher than those in the initial $\mathrm{BC}$, suggesting that biocrude organics have better solubility in MF than inorganic-bound compounds of BC. Since the inorganic content of MF is negligible, with all the measured concentrations under the calibration or the limit of detection of the instrument, it is straightforward to ascribe the presence of inorganics in the blends only to the biocrude's contribution.

Table 3. Main inorganic elements of SS biocrude, MF and blend samples. (in bracket abs. std. dev.).

\begin{tabular}{ccccccc}
\hline ID of Sample & Fe (ppm) & Ca (ppm) & $\mathbf{P}(\mathbf{p p m})$ & $\mathbf{K}(\mathbf{p p m})$ & Si (ppm) & Al (ppm) \\
\hline SS biocrude & $716(3.5)$ & $202(1.9)$ & $196(10.6)$ & $106(1.9)$ & $90(0.5)$ & 41 \\
MF & u.d.l. & u.d.l. & u.c.l. & u.d.l. & u.d.l. & u.d.l. \\
Blend A & $54(2.5)$ & $27(1.8)$ & u.c.l. & u.d.l. & $14(0.7)$ & 9 \\
Blend B & $75(0.5)$ & $16(2.3)$ & u.c.l & u.d.l. & u.d.l. & 9 \\
Blend B-residue & $1048(15.8)$ & $720(9.8)$ & $531(16.7)$ & $379(5.4)$ & $232(1.3)$ & 209 \\
\hline
\end{tabular}

u.d.l. = under detection limit; u.c.l. = under calibration limit (10 ppm).

Based on the results reported in Table 3, one can observe that the concentration of inorganics in Blend A and B are comparable and unrelated to the concentration of SS BC in blend, suggesting also that some solubility limit was attained for each of those elements in the MF.

\subsection{Blend Compliance with MF International Standards}

In order to be commercially used in marine diesel engines, a residual marine fuel must comply with the international standard ISO 8217:2017. In this work, we focused our analysis on the elemental composition, notably $\mathrm{C}, \mathrm{H}, \mathrm{N}, \mathrm{O}$, and $\mathrm{S}$, the content of ash and inorganics ( $\mathrm{Va}, \mathrm{Na})$, and the acidity.

Considering the results of the mixing experiments discussed in Section 3.1 and the inferred blending limit, the analyses reported hereafter were performed only on the blend with 10 wt.\% BC concentration (Blend A).

The acidic compounds in fuels could occasionally cause accelerated damage to marine diesel engines, in particular to the fuel injection equipment. TAN gives an indication of the amount of acidic compounds and the ISO 8217:2017 prescribes a limit for residual marine fuel at $2.5 \mathrm{mg} \mathrm{KOH} / \mathrm{g} \mathrm{[25].}$

The TAN of the MF sample was $0.32 \mathrm{mg} \mathrm{KOH} / \mathrm{g}$ (Table 1); with the addition of $10 \mathrm{wt} . \% \mathrm{SS}$ biocrude, TAN increased to $1.43 \mathrm{mg} \mathrm{KOH} / \mathrm{g}$, however still below the prescribed limit [25] despite the 4.5-fold increase.

Another relevant characteristic for a commercial fuel is the ash content. All residual fuels contain metallic species already present in the crude oil feedstock or introduced 
from external sources. During combustion, these metals are converted to ashes, mainly composed of solid particles of oxides, sulfates and other complex compounds. The high temperature achieved during the combustion could cause melting of these particles; these fluid ashes can thus adhere to the surface of the combustion chamber, generating a set of negative impacts, damages and corrosion [25]. The melting temperature (often referred as "stiction temperature") greatly varies depending on ash constituents; it is the lowest in the case of ashes rich in vanadium and/or sodium. Vanadium is a natural component of every crude oil and therefore it can be found in most fuel oils, while sodium is generally related to sea water contamination, because it is not normally present in an oil-soluble form [41]. ISO 8217 sets a limit of $350 \mathrm{mg} \mathrm{kg}^{-1}$ of vanadium and $100 \mathrm{mg} \mathrm{kg}^{-1}$ of sodium to comply with the specifications of grade ISO-F-RMG 380 [25]. Moreover, The International Council on Combustion Engines (CIMAC) suggests to avoid fuel oil with vanadium content above $150 \mathrm{mg} \mathrm{kg}^{-1}$ and with sodium in an oil-soluble form [41].

For both blends (Blend A and B), the measured vanadium, sodium and silicon + aluminum contents were below those suggested by CIMAC and prescribed by ISO 8217 .

Moreover, the norm also prescribes a limit of $0.1 \mathrm{wt} . \%$ ash in the fuel, but the measured ash content in Blend A was $0.137 \mathrm{wt.} \%$ (Std. dev. = 0.05); it was almost entirely due to the biocrude addition because the MF resulted to be substantially ash free (Table 1). The measured sulphur content of MF used in these experiments was $0.38 \mathrm{wt} . \%$ (as given in Table 1); the addition $10 \mathrm{wt} . \% \mathrm{BC}$ slightly increased this figure to $0.47 \mathrm{wt} . \%$ (abs. stdev $=0.005$ ) remaining below the limit imposed by IMO of $0.5 \mathrm{wt} . \%$.

\subsection{Summary of BLENDED MARITIME Fuel with International Standards}

The analyses performed on Blend A show that the addition of the $10 \%$ biocrude in MF is a feasible option to reduce the carbon footprint of marine transportation, in particular in large ocean-going ships, while meeting the specifications for the set of characteristics here investigated. The reproducibility of the analytical results (low standard deviation) confirmed that the obtained blend has a homogeneous composition that could promote its adoption in marine diesel engine.

Blend A falls within the prescribed ISO 8217:2017 limits for TAN and contents of sulphur, vanadium, sodium and silicon + aluminum. Only the ash content is slightly above the limit, a minor issue that given the impact potential of introducing SS BC in the marine sector is well worth investigating, and could be addressed e.g., by improved biocrude filtration, demineralization, or the adaptation of HTL parameters steps prior to blending with MF.

The experiments herein reported have proven the possibility of blending SS biocrude with MF, without any previous upgrade, and obtaining a blend that complies with the basic quality parameters for the original fuel under ISO 8217:2017 and identifying a blending limit between $14-16$ wt.\%.

\subsection{FT-IR}

The FT-IR spectra of marine fuel, biocrude and blends were also investigated.

Marine fuel (Figure 4) mostly presents $\mathrm{CH}$ signals typical of alkanes, as the $\mathrm{C}-\mathrm{H}$ in $\mathrm{CH}_{2}$ vibration and $\mathrm{CH}$ in $\mathrm{CH}_{3}$ stretching [42,43] around 2920, 2960 and $2860 \mathrm{~cm}^{-1}$, and the signals at 1375 and $1460 \mathrm{~cm}^{-1}$ related to the $\mathrm{C}-\mathrm{H}$ bond in $\mathrm{CH}_{3}$ bending [43]. However, a small band around $1600 \mathrm{~cm}^{-1}$ points out the presence of $\mathrm{C}=\mathrm{C}$ stretching ascribed to aromatics compounds such as polycyclic aromatics [44]. 


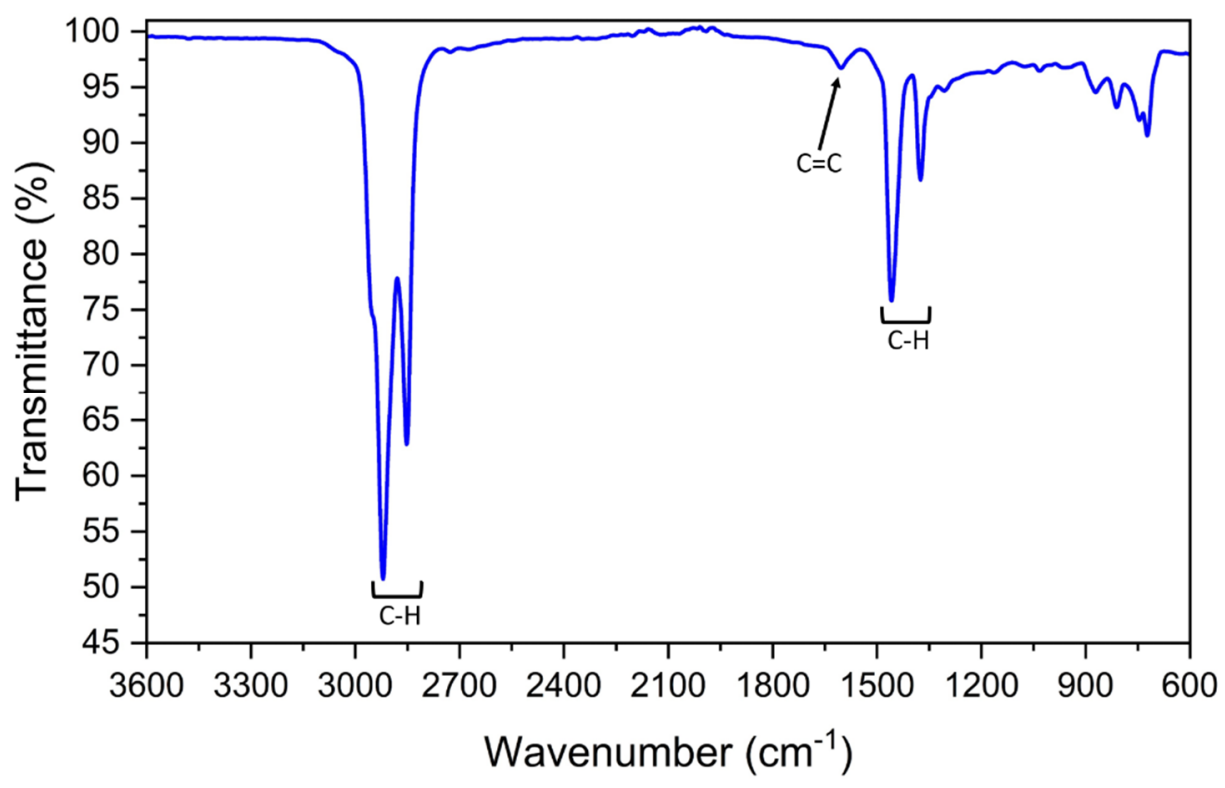

Figure 4. FT-IR spectrum of marine fuel.

The SS biocrude spectrum (Figure 5) also presents signals in the aliphatic $\mathrm{C}-\mathrm{H}$ range (2920-2860, 1460 and $1380 \mathrm{~cm}^{-1}$ [43]) which were attributed to lipids or hydrocarbons presence, but oxygenated groups are also detected, such as the $\mathrm{OH}$ signal around $3300 \mathrm{~cm}^{-1}$, also ascribed to N-H stretching [45], or the peak around $1700 \mathrm{~cm}^{-1}$ which highlights the presence of $\mathrm{C}=\mathrm{O}$ groups [43] probably related to carboxylic acids or amides. The signals around $1600-1660 \mathrm{~cm}^{-1}$, ascribed to the presence of amides and nitrogen heterocyclic compounds in the biocrude [43-47].

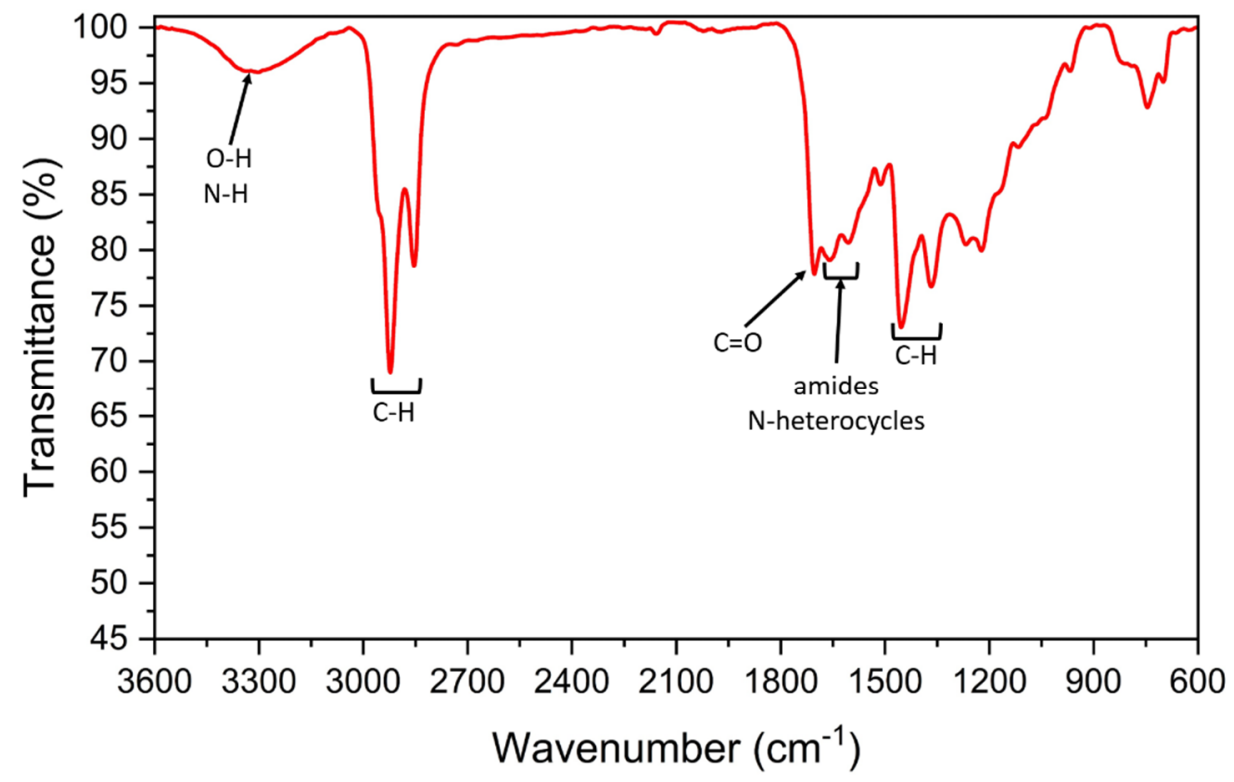

Figure 5. FT-IR spectrum of SS biocrude.

Both blends (Blend A and B) show the peculiar peaks of the SS crude, such as the 3300 and $1700 \mathrm{~cm}^{-1}$ signals related to the oxygenated and nitrogen compounds of the biocrude (i.e., fatty acids, and primary amides $[43,48]$ ) as well as the signals around $1600-1660 \mathrm{~cm}^{-1}$, most likely amides and pyrrolic compounds [38,43,45], but with higher C-H signals at 1460 and $1375 \mathrm{~cm}^{-1}$, typical of the MF, therefore-as expected—showing both of the feedstock's 
chemical group's characteristics (Figure 6) and thus confirming, within the limit of the qualitative nature of the analysis, the formation of a mixture.

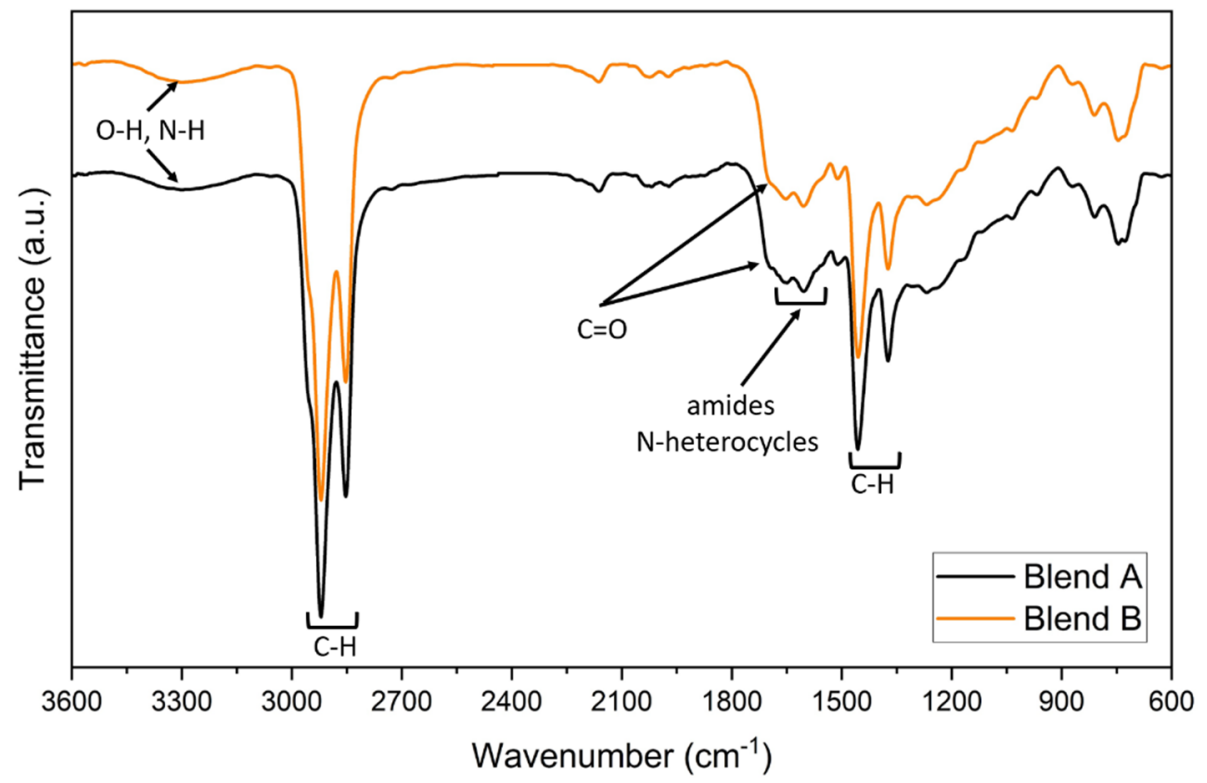

Figure 6. FT-IR spectrum of $10 \%$ and $20 \%$ SS biocrude blends.

The assessment of the cause-effect relationship between FT-IR spectra and blends' properties was not, on the other hand, part of the intended scope of the work, and the author did not found evidence of a correlation between FT-IR spectra, which are qualitative, and the chemical/physical properties of the mixture that could be measured/analyzed with the available volume of sample.

\section{Conclusions}

The miscibility of SS biocrude in residual marine fuel (low-S RMG per ISO 8217) was investigated using analytical methods (elemental analysis, FT-IR) and two blends were prepared at $10 \mathrm{wt} . \%$ (Blend A) and $20 \mathrm{wt} . \%$ (Blend B) nominal concentration of BC in blend. Blend A resulted in a homogeneous, mobile black liquid; the presence of suspended particles or agglomerates could not be detected, suggesting that most of the biocrude was dispersed in the MF.

As regards the case of Blend $B$, notwithstanding the thorough mixing of biocrude and $\mathrm{MF}$, the formation of agglomerates could be identified, thus indicating an incomplete blending of $\mathrm{BC}$ in the MF. The results of elemental analysis on the homogeneous samples confirmed the presence of biocrude in the two blends; in fact, the carbon content slightly decreased, while nitrogen and oxygen contents increased. The effective mixing of these two substances was also confirmed by the FT-IR spectra. The two blends show peculiar peaks of the SS BC related to oxygenated and nitrogen compounds with a higher C-H signal at 1460 and $1375 \mathrm{~cm}^{-1}$, typical of the MF. From the analytical determinations carried out in this study, it was possible to calculate that the blending limit of this specific sample of SS BC in MF was between 14-16 wt.\%.

As regards the selected group of chemical characteristics analysed in this work, Blend A met the basic quality parameters (TAN, inorganics and sulphur content) set forth by international standard ISO 8217:2017 for grade ISO-F-RMG 380 marine fuel, thus showing a promising route for bio-component introduction in commercial fossil fuels for maritime applications. 


\begin{abstract}
Author Contributions: Conceptualization, A.M.R. and D.C.; methodology, A.M.R.; resources, A.M.R.; data curation, A.M.R. and D.C.; writing-original draft preparation, A.M.R.; writing-review and editing, A.M.R. and D.C.; visualization, A.M.R.; supervision, D.C.; project administration, A.M.R.; funding acquisition, D.C. All authors have read and agreed to the published version of the manuscript.

Funding: This research received no external funding.

Institutional Review Board Statement: Not applicable.

Informed Consent Statement: Not applicable.

Data Availability Statement: Data available upon request to the corresponding Author.

Acknowledgments: The authors gratefully acknowledge Arturo di Fraia (RE-CORD) for the execution of the hydrothermal liquefaction test and mixing trials, Francesca Tozzi (RE-CORD) for her support in the statistical analyses, and Giulia Lotti (RE-CORD) and Lorenzo Bettucci (RE-CORD) for the chemical analyses. The provision of the marine fuel sample from Daniele Bianchi (ENI) and Irene Rapone (ENI) is also kindly appreciated.
\end{abstract}

Conflicts of Interest: The authors declare no conflict of interest.

\title{
References
}

1. Proposal for a Regulation of the European Parliament and of the Council on the Use of Renewable and Low-Carbon Fuels in Maritime Transport and Amending Directive 2009/16/EC; COM/2021/562 Final. Available online: https:/ / eur-lex.europa.eu/ legal-content/EN/TXT/?uri=CELEX\%3A52021PC0562 (accessed on 1 June 2021).

2. EUROSTAT Passengers Embarked and Disembarked in All Ports by Direction-Annual Data (MAR_PA_AA). Available online: https:/ / ec.europa.eu/eurostat/databrowser/bookmark/e595bed3-7558-4560-b69a-acf0ccf490e7?lang=en (accessed on 29 September 2021).

3. Regulation (EU) $2021 / 1119$ of the European Parliament and of the Council Establishing the Framework for Achieving Climate Neutrality and Amending Regulations (EC) No 401/2009 and (EU) 2018/1999 ('European Climate Law'); PE/27/2021/REV/1, OJ L 243, 9.7.2021, pp. 1-17. Available online: https:/ / eur-lex.europa.eu/legal-content/EN/TXT/?uri=CELEX\%3A32021R1119 (accessed on 1 June 2021).

4. Dimitriadis, A.; Bezergianni, S. Hydrothermal liquefaction of various biomass and waste feedstocks for biocrude production: A state of the art review. Renew. Sustain. Energy Rev. 2017, 68, 113-125. [CrossRef]

5. Castello, D.; Pedersen, T.; Rosendahl, L. Continuous Hydrothermal Liquefaction of Biomass: A Critical Review. Energies 2018, 11, 3165. [CrossRef]

6. Ramirez, J.A.; Brown, R.J.; Rainey, T.J. A review of hydrothermal liquefaction bio-crude properties and prospects for upgrading to transportation fuels. Energies 2015, 8, 6765-6794. [CrossRef]

7. Wang, S.; Zhao, S.; Cheng, X.; Qian, L.; Barati, B.; Gong, X.; Cao, B.; Yuan, C. Study on two-step hydrothermal liquefaction of macroalgae for improving bio-oil. Bioresour. Technol. 2021, 319, 124176. [CrossRef]

8. United Nations-Department of Economic and Social Affairs-Population Division World Population Prospects 2019: Highlights (ST/ESA/SER.A/423). Available online: https://population.un.org/wpp/Publications/Files/WPP2019_Highlights.pdf (accessed on 27 October 2021).

9. Campo, G.; Cerutti, A.; Lastella, C.; Leo, A.; Panepinto, D.; Zanetti, M.; Ruffino, B. Production and Destination of Sewage Sludge in the Piemonte Region (Italy): The Results of a Survey for a Future Sustainable Management. Int. J. Environ. Res. Public Health 2021, 18, 3556. [CrossRef] [PubMed]

10. Istituto Superiore per la Protezione e la Ricerca Ambientale (ISPRA). Rapporto Rifiuti Speciali-Edizione 2021-Dati di Sintesi345/2021; ISPRA: Rome, Italy, 2021. Available online: https:/ / www.isprambiente.gov.it/it/pubblicazioni/rapporti/rapportorifiuti-speciali-edizione-2021 (accessed on 1 June 2021).

11. Shah, A.A.; Toor, S.S.; Conti, F.; Nielsen, A.H.; Rosendahl, L.A. Hydrothermal liquefaction of high ash containing sewage sludge at sub and supercritical conditions. Biomass Bioenergy 2020, 135, 105504. [CrossRef]

12. Qian, L.; Wang, S.; Savage, P.E. Fast and isothermal hydrothermal liquefaction of sludge at different severities: Reaction products, pathways, and kinetics. Appl. Energy 2020, 260, 114312. [CrossRef]

13. Shah, A.A.; Toor, S.S.; Seehar, T.H.; Nielsen, R.S.; Nielsen, A.H.; Pedersen, T.H.; Rosendahl, L.A. Bio-crude production through aqueous phase recycling of hydrothermal liquefaction of sewage sludge. Energies 2020, 13, 493. [CrossRef]

14. Xu, D.; Lin, G.; Liu, L.; Wang, Y.; Jing, Z.; Wang, S. Comprehensive evaluation on product characteristics of fast hydrothermal liquefaction of sewage sludge at different temperatures. Energy 2018, 159, 686-695. [CrossRef]

15. Mortensen, P.M.; Grunwaldt, J.-D.; Jensen, P.A.; Knudsen, K.G.; Jensen, A.D. A review of catalytic upgrading of bio-oil to engine fuels. Appl. Catal. A Gen. 2011, 407, 1-19. [CrossRef]

16. Castello, D.; Haider, M.S.; Rosendahl, L.A. Catalytic upgrading of hydrothermal liquefaction biocrudes: Different challenges for different feedstocks. Renew. Energy 2019, 141, 420-430. [CrossRef] 
17. Jarvis, J.M.; Albrecht, K.O.; Billing, J.M.; Schmidt, A.J.; Hallen, R.T.; Schaub, T.M. Assessment of Hydrotreatment for Hydrothermal Liquefaction Biocrudes from Sewage Sludge, Microalgae, and Pine Feedstocks. Energy Fuels 2018, 32, 8483-8493. [CrossRef]

18. Chiaberge, S.; Siviero, A.; Passerini, C.; Pavoni, S.; Bianchi, D.; Haider, M.S.; Castello, D. Co-processing of Hydrothermal Liquefaction Sewage Sludge Biocrude with a Fossil Crude Oil by Codistillation: A Detailed Characterization Study by FTICR Mass Spectrometry. Energy Fuels 2021, 35, 13830-13839. [CrossRef]

19. Paulauskiene, T.; Bucas, M.; Laukinaite, A. Alternative fuels for marine applications: Biomethanol-biodiesel-diesel blends. Fuel 2019, 248, 161-167. [CrossRef]

20. IHS Markit. IMO 2020: What Every Shipper Needs to Know; HIS Markit: London, UK, 2019. Available online: https:/ www. seaburycapital.com/wp-content/uploads/2019/02/GeminiWhitepaper2019-v2b-abbb.pdf (accessed on 1 June 2021).

21. Vermiere, M.B. Everything You Need to Know about Marine Fuels; Chevron Product Engineering Department: San Ramon, CA, USA, 2021; Available online: https://www.chevronmarineproducts.com/content/dam/chevron-marine/Brochures/Chevron_ EverythingYouNeedToKnowAboutFuels_v3_1a_DESKTOP.pdf (accessed on 1 June 2021).

22. Basha, S.I.; Aziz, A.; Maslehuddin, M.; Ahmad, S.; Hakeem, A.S.; Rahman, M.M. Characterization, Processing, and Application of Heavy Fuel Oil Ash, an Industrial Waste Material-A Review. Chem. Rec. 2020, 20, 1568-1595. [CrossRef]

23. Heavy Fuel Oil (HFO). Available online: https://www.marquard-bahls.com/en/news-info/glossary/detail/term/heavy-fueloil-hfo.html (accessed on 1 October 2021).

24. Marine Gasoil (MGO). Available online: https://www.marquard-bahls.com/en/news-info/glossary/detail/term/marinegasoil-mgo.html (accessed on 1 October 2021).

25. International Organization for Standardization. ISO 8217-Petroleum Products_Fuels (Class F)—Specifications of Marine Fuels; ISO: Geneva, Switzerland, 2017.

26. Corbett, J.J.; Winebrake, J.J.; Green, E.H.; Kasibhatla, P.; Eyring, V.; Lauer, A. Mortality from Ship Emissions: A Global Assessment. Environ. Sci. Technol. 2007, 41, 8512-8518. [CrossRef] [PubMed]

27. Directive (EU) 2016/802 of the European Parliament and of the Council of 11 May 2016-Relating to a Reduction in the Sulphur Content of Certain Liquid Fuels; OJ L 132, 21.5.2016, p. 58-78. Available online: https://eur-lex.europa.eu/legal-content/en/ TXT/?uri=CELEX:32016L0802 (accessed on 1 June 2021).

28. International Maritime Organization. IMO 2020-Sulfur FAQ; IMO: London, UK, 2020. Available online: http://www.imo.org/en/ MediaCentre/HotTopics/Documents/Sulphur2020infographic2page.pdf (accessed on 1 June 2021).

29. Corbett, J.J.; Winebrake, J.J.; Carr, E.W.; Jalkanen, J.-P.; Johansson, L.; Prank, M.; Sofiev, M. Health Impacts Associated with Delay of MARPOL Global Sulphur Standards; MEPC 70/INF.34; Finnish Meteorological Institute: Helsinki, Finland, 2016.

30. Garaniya, V.; McWilliam, D.; Goldsworthy, L.; Ghiji, M. Extensive chemical characterization of a heavy fuel oil. Fuel 2018, 227, 67-78. [CrossRef]

31. Goldsworthy, L. Computational Fluid Dynamics Modelling of Residual Fuel Oil Combustion in the Context of Marine Diesel Engines. Int. J. Engine Res. 2006, 7, 181-199. [CrossRef]

32. Rizzo, A.M.; Dell'Orco, S.; Miliotti, E.; Chiaramonti, D. Design, Commissioning And Start-Up of a New Hydrothermal Liquefaction Continuous Pilot Unit. Chem. Eng. Trans. 2020, 80, 367-372. [CrossRef]

33. Rahman, T.; Jahromi, H.; Roy, P.; Adhikari, S.; Hassani, E.; Oh, T.-S. Hydrothermal liquefaction of municipal sewage sludge: Effect of red mud catalyst in ethylene and inert ambiences. Energy Convers. Manag. 2021, 245, 114615. [CrossRef]

34. Conti, F.; Toor, S.S.; Pedersen, T.H.; Seehar, T.H.; Nielsen, A.H.; Rosendahl, L.A. Valorization of animal and human wastes through hydrothermal liquefaction for biocrude production and simultaneous recovery of nutrients. Energy Convers. Manag. 2020, 216, 112925. [CrossRef]

35. Nazari, L.; Yuan, Z.; Souzanchi, S.; Ray, M.B.; Xu, C. Hydrothermal liquefaction of woody biomass in hot-compressed water: Catalyst screening and comprehensive characterization of bio-crude oils. Fuel 2015, 162, 74-83. [CrossRef]

36. Barnés, M.C.; de Visser, M.M.; van Rossum, G.; Kersten, S.R.A.; Lange, J.-P. Liquefaction of wood and its model components. J Anal. Appl. Pyrolysis 2017, 125, 136-143. [CrossRef]

37. Li, H.; Zhu, Z.; Lu, J.; Watson, J.; Kong, D.; Wang, K.; Zhang, Y.; Liu, Z. Establishment and performance of a plug-flow continuous hydrothermal reactor for biocrude oil production. Fuel 2020, 280, 118605. [CrossRef]

38. Prado, G.H.C.; Rao, Y.; de Klerk, A. Nitrogen Removal from Oil: A Review. Energy Fuels 2017, 31, 14-36. [CrossRef]

39. Laredo, G. Nitrogen compounds characterization in atmospheric gas oil and light cycle oil from a blend of Mexican crudes. Fuel 2002, 81, 1341-1350. [CrossRef]

40. Zhai, Y.; Chen, H.; Xu, B.; Xiang, B.; Chen, Z.; Li, C.; Zeng, G. Influence of sewage sludge-based activated carbon and temperature on the liquefaction of sewage sludge: Yield and composition of bio-oil, immobilization and risk assessment of heavy metals. Bioresour. Technol. 2014, 159, 72-79. [CrossRef]

41. The International Council on Combustion Engines. Recommendations Regarding Fuel Quality for Diesel Engines; The International Council on Combustion Engines: Frankfurt, Germany, 2003. Available online: https://www.cimac.com/cms/upload/ Publication_Press/Recommendations/Recommendation_21_rev1.pdf (accessed on 1 June 2021).

42. Guo, A.; Ren, Z.; Tian, L.; Wang, Z.; Li, K. Characterization of molecular change of heavy oil under mild thermal processing using FT-IR spectroscopy. J. Fuel Chem. Technol. 2007, 35, 168-175. [CrossRef] 
43. Vardon, D.R.; Sharma, B.K.; Scott, J.; Yu, G.; Wang, Z.; Schideman, L.; Zhang, Y.; Strathmann, T.J. Chemical properties of biocrude oil from the hydrothermal liquefaction of Spirulina algae, swine manure, and digested anaerobic sludge. Bioresour. Technol. 2011, 102, 8295-8303. [CrossRef]

44. Kowalski, M.; Kowalska, K.; Wiszniowski, J.; Turek-Szytow, J. Qualitative analysis of activated sludge using FT-IR technique. Chem. Pap. 2018, 72, 2699-2706. [CrossRef] [PubMed]

45. Wang, T.; Zhai, Y.; Zhu, Y.; Peng, C.; Xu, B.; Wang, T.; Li, C.; Zeng, G. Influence of temperature on nitrogen fate during hydrothermal carbonization of food waste. Bioresour. Technol. 2018, 247, 182-189. [CrossRef]

46. Xu, Z.-X.; Xu, L.; Cheng, J.-H.; He, Z.-X.; Wang, Q.; Hu, X. Investigation of pathways for transformation of N-heterocycle compounds during sewage sludge pyrolysis process. Fuel Process. Technol. 2018, 182, 37-44. [CrossRef]

47. Wei, F.; Cao, J.-P.; Zhao, X.-Y.; Ren, J.; Wang, J.-X.; Fan, X.; Wei, X.-Y. Nitrogen Evolution during Fast Pyrolysis of Sewage Sludge under Inert and Reductive Atmospheres. Energy Fuels 2017, 31, 7191-7196. [CrossRef]

48. Grube, M.; Lin, J.G.; Lee, P.H.; Kokorevicha, S. Evaluation of sewage sludge-based compost by FT-IR spectroscopy. Geoderma 2006, 130, 324-333. [CrossRef] 